\title{
La ética desde el feminismo. Notas sobre la 'diferencia'
}

\author{
MARfA HERRERA LIMA \\ Instituto de Investigaciones Filosóficas, UNAM, México
}

Una de las tareas que se ha propuesto la reflexión feminista en cuestiones filosóficas, es la crítica al universalismo ético en sus formulaciones clásicas. Esta reflexión tiene en común con otras formas de crítica a las Éticas filosóficas de corte ilustrado, su interés por el análisis contextual, y la reconstrucción histórica de nociones clave para la conceptualización de la experiencia moral: problemas tales como los relativos a la constitución de la identidad de los sujetos morales, o la configuración social e histórica de lo que, siguiendo a Charles Taylor,' podemos llamar el 'espacio moral' desde el cual los sujetos proponen las distinciones pertinentes a la legitimidad o corrección moral de sus acciones. En esta tarea, el pensamiento feminista ha desempeñado un papel importante al destacar la diferencia de sexos como un eje indispensable en la comprensión de la historia misma de las döctrinas morales.

Podríamos señalar, por ejemplo, la recuperación, bajo una nueva luz, de contribuciones como la de lévi-Strauss en relación con el sentido del carácter fundador para la cultura del tabú del incesto. Como lo propone Celia Amorós: invertir los términos de una concepción 'naturalista' de la relación entre hombre y mujer, y verla como una relación «mediata y cultural», que si bien tiene a la naturaleza como referencia, siempre está «mediada de alguna forma por los pactos que los hombres establecen entre sí como 'dadores' y 'tomadores' de mujeres».?
Además de la 'desnaturalización' de las relaciones entre los sexos, esta inversión tiene como consecuencia el identificar los inicios de la exclusión de las mujeres del dominio de la moralidad. Al ser concebidas a partir de su relación con los hombres, ni la relación entre hombre y mujer es una verdadera relación, ya que es definida y condicionada por las relaciones entre hombres, ni la mujer, por consiguiente, puede ser considerada como un agente o sujeto autónomo. Habría ciertamente otras líneas de investigación emprendidas por pensadoras feministas respecto de problemas de la filosofia moral. Entre las más conocidas, podemos mencionar el trabajo de Carol Gilligan ${ }^{3}$ sobre las diferencias en la constitución y desarrollo de la conciencia moral en los miembros de cada sexo. Además de un buen número de trabajos que han derivado de esas investigaciones, y que atribuyen a las diferencias en la socialización, la configuración de lo que Marilyn Friedman llama proyectos morales diferenciados, ${ }^{4}$ esto es, formas distintas de estructurar las relaciones con otros a partir de la experiencia social de los sujetos.

No obstante, podríamos observar un hilo conductor detrás de una parte considerable de la reflexión feminista sobre la moralidad: éste tendría que ver con la recuperación de las dimensiones concretas de la experiencia moral, con un énfasis en las prácticas, la formación de una identidad ( $y$ del carácter y las 'virtudes') y de una idea de 
sí mismo, de la clase de persona que se quiere ser (o de aquello que no queremos ser), que se oponen a la reducción formalista de la ética a problemas de justificación de las normas morales o a una teoria de la justicia. Dentro de una amplia variedad de matices teóri$c o s$, pareceria haber un interés más o menos generalizado por recuperar al 'otro concreto', como lo llama Seyla Benhabib' y tomar distancia, tanto de las abstracciones metafísicas de el o lo 'Otro', como de las formulaciones de un universalismo vacío. Celia Amorós expresó esto hace algún tiempo como la necesidad de la sdenuncia de la ficción de universalidad». $\mathrm{Si}$ bien, esto no supone, ni para ella, ni para un buen número de nosotras, la renuncia a una utopía igualitaria. Se trataría, más bien, de recuperar, transformándolo, el ideal ilustrado de una igualdad que no suprimiera o neutralizara artificiosamente las diferencias, sino que hiciera posible el ejercicio de la libertad a todos los sujetos. Una igualdad que en todo caso se asume como 'punto de llegada' y no como 'punto de partida' del aciuar moral.

Pero una vez reconocidas nuestras aspiraciones igualitarias y universalistas, podemos de nuevo formular la pregunta acerca de la diferencia, o mejor, las diferencias, entre las que la diferencia de género ocupa un lugar privilegiado y paradigmático.

Una sugerencia inquietante -como la duda sobre la irracionalidad del emotivismo que habría expresado alguna vez Mclntyre-7 podría desprenderse de ese haber sido definidas por la exclusión. De ese tener que depender aún para la crítica al orden establecido de «un imaginario no compartido por toda la especie» como lo señala Amelia Valcárcel, ${ }^{8}$ o del aceptar con Foucault que la Etica, desde sus inicios en la antiguiedad clásica, fue una ética masculina, hecha por $\mathrm{y}$ para los varones. ${ }^{9}$ Una inquietud que se manifiesta en las oscilaciones entre la orientación a la 'igualdad' o la 'diferencia' de las propuestas feministas, o en la ambigüedad frente a la tradición liberal del feminismo radical, como el de Iris Marion Young, ${ }^{10}$ que pondría en duda nuestra membrecía -en cualquier caso tardía y anómala- a la ciudadanía completa. Un caso reciente, que hace patente las limitaciones de ideas como la de la 'imparcialidad' de la ley, es el de la disputa entre Anita Hill y el candidato a juez, en espera de su confirmación, Clarence Thomas. En un penetrante analisis de este caso, Nancy Fraser ${ }^{11}$ ve cómo las relaciones de poder/autoridad lograron establecer, de manera desfavorable para ella, las fronteras entre aquello que debía abrirse al escrutinio público y lo que en cambio debía preservarse como 'privado', así como también habría designado a aquellos que tendrían que servir de salvaguardia a dichas fronteras. Si algo podemos aprender de este caso es que la complejidad de estos procesos nos impide reducirlos a explicaciones simplificadoras, por ejemplo, en términos de un discurso de los 'dominantes' y otro de los 'dominados'. No sólo porque allí se entrecruzaron $\mathrm{mu}$ chas formas de desigualdad (sexuales, étnicas) y de poder (de nuevo sexual y étnico pero a la vez institucional y jerárquico) sino porque estos mismos discursos se utilizaron de manera perversa, por ejemplo apelando a sentimientos anti-racistas en defensa del juez Thomas pero no de ella. Además de otras conclusiones interesantes en torno a la idea del espacio público que ofrece este trabajo, nos recuerda el peligro de acudir a una defensa de la 'diferencia' por la diferencia misma, ya que esto puede sumergirnos 
en un pantano de confusiones: ¿se defiende el derecho de las (los) desiguales a ocupar un sitio comparable al que disfrutan las identidades sociales dominantes?, ¿es una defensa de la legitimidad de formas de vida y de expresión linguístico/culturales distintas al modelo dominante?, o ¿es acaso, y de qué manera, una forma de complicidad con un régimen de poder?

Parecería que cuando intentamos ponerlas a prueba en instancias tan complejas como la arriba mencionada, ni las nociones liberales de tolerancia ni la simple postulación de la 'diferencia' resultan suficientes. La tolerancia es por supuesto necesaria como respeto a la disidencia o a lo diferente, visto un tanto a distancia, pero no puede ser una forma de resolución de conflictos del tipo indicado. O pensemos, por ejemplo, en la caracterización que hace Lyotard de la diferencia'. En un libro suyo que lleva ese título: ${ }^{i 2}$ nos propone entender la diferencia como un conflicto entre géneros o discursos heterogéneos, pero como un conflicto que no puede resolverse por los medios tradicionales ya que carecemos de «una regla de juicio aplicable a las dos argumentaciones». No se trata de una forma ordinaria de litigio ya que éste presupone acuerdo en los términos de la disputa, una cierta homogeneidad en los discursos de los contendientes, y podríamos agregar nosotros, una cierta igualdad en cuanto al acceso a recursos argumentativos revestidos de la autoridad que confiere una posición de poder. En términos de la formulacion de Lyotard, podríamos ver la theterogeneidad' de los discursos en la forma en que cada uno definía el mismo hecho (el acoso sexual) o bien como una forma inocente de camaradería' asignada al territorio de la 'normalidad', o bien como un 'abuso de poder, como una ofensa y aun una forma de conducta patológica. ${ }^{13}$ Lyotard tendría razón al afirmar que no podemos resolver esta clase de conflictos intentando una falsa disolución o neutralización de las diferencias, pero ¿qué salida nos ofrece, para evitar, en sus palabras, el 'abismo de la heterogeneidad'?

Lyotard describe algunas formas de supresión de las diferencias $-o$ de $s u$ asimilación en una narrativa unificada- que no tienen que suponer, por otra parte, una forma de 'solución' a conflictos que pudieran surgir entre las formas de discurso o narraciones particulares así unificadas. Se puede pensar, por ejemplo, en la apropiación que para sus fines realizan los mitos, que equivale a una supresión simbólica (o una 'neutralización' en palabras de Lyotard) de las diferencias. Podriamos pensar también en las diferencias 'suspendidas' por los 'pactos' o 'treguas' como acuerdos de paz eatre contendientes, y en las diferencias 'olvidadas' en los relatos que les imponen un fin unificador. O tambićn en las formas modernas de supresión de la particularidad, en el tipo de relato universalista que suprime los 'nombres' de una gran Historia de la Humanidad. Para Lyotard estos últimos conservan de sus antecesores religiosos el forjarse en torno a una idea del futuro 'como redención' ${ }^{14}$

En estos ejemplos, se apela a la narrativa como una forma más adecuada de incluir la particularidad de relatos heterogéneos, más que intentar ofrecer alguna forma de conciliarlos. Pareceria que las formas de 'suspensión' temporal de las diferencias (en los 'pactos' o acuerdos neutralizadores) sólo podrían realizarse en la forma de acuerdos locales, sobre cuestiones puntuales, sin pretender ofrecer alguna formula de alcances más 
generales. Aún en estos casos, no parecería haber ningún criterio o guía para alcanzar un acuerdo. Pero no es sólo la ausencia de alguna idea de 'consenso' posible lo que presenta un problema, ésta es de hecho una consecuencia del supuesto de la heterogeneidad de los discursos, sino más bien la reducción implícita a la dimensión estratégica en la interacción social. No es sólo que los "juegos de lenguaje' plurales queden 'abiertos' de manera lúcida a una interacción libre, sino que quedan igualmente abiertos a la intervención del poder: ¿quién, o entre quiénes se deciden los 'pactos?? ¿qué diferencias aceptan ser -provisionalmente- neutralizadas? (por ejemplo, la diferencia racial en el caso del juez Thomas, en aras de una alianza entre varones, o entre superiores jerárquicos, etc.), ¿cuáles, en cambio, aparecen como un 'estigma'?

Como sugeríamos antes, al agregar a la cuestión de la heterogeneidad de las discursos (que subsiste como un problema real) la disparidad de estos lenguajes en cuanto a su relación con el poder, o en términos más precisos, su posición de mayor o menor autoridad' o reconocimiento y aceptación social derivada de su posición en el entramado de relaciones sociales $y$ políticas, lo que está en juego es algo más que la supresión de los meta-relatos falsamente unificadores. Si el ignorar u 'olvidar' las diferencias asimilándolas a grandes relatos unificadores o neutralizándolas en lenguajes asépticos que se pretenden universales, es una forma de hacer violencia a las diferencias reales de los sujetos particulares (en tanto que individuos o miembros de colectividades marginales, por su número o por su condición frente al poder) la simple postulación de una pluralidad de voces narrativas no toca siquiera el problema de otras formas de violencia que se ejercen entre ellas.

El feminismo no puede dejar intocadas estas formas de violencia. Sino que, citando de nuevo a Amelia Valcárcel, debe dirigirse a ese "nuevo continente exploratorio de nuestro siglo, que guardan pensamiento y poder». ${ }^{15}$ Una de las reflexiones más ricas y profundas en torno a este tema es sin duda la de Michel Foucault. En especial, sus últimos trabajos y la Historia de la sexualidad.

No quisiera, sin embargo, detenerme en la discusión interna a la obra de Foucault, por ejemplo, en cuanto a los cambios en su teoría del poder a lo largo de su obra, sino solamente extraer algunas consideraciones interesantes para nuestro tema. Para ello quiero citarlo con cierta extensión, cuando caracteriza al poder en una entrevista, realizada en 1984 , y más tarde revisada por él mismo para su publicación:

Cuando uno habla de 'poder', la gente piensa habitualmente en una estructura politica, en una clase dominante, el señor frente al esclavo, etc. No es eso todo lo que yo pienso cuando hablo de relaciones de poder'. Quiero decir que en las relaciones humanas, cualesquiera que sean - tanto si es un asunto de comunicación verbal [...] o de amor, o una relación institucional o económica- el poder está siempre presente: me refiero a las relaciones en las que se quiere dirigir la conducta de otro [...] Estas relaciones son cambiantes, neversibles $y$ comprensibles [...] Ahora bien, hay efectivamente situaciones de dominación. En muchos casos las relaciones de poder están fijadas de tal manera que son asimétricas a perpetuidad y el margen de libertad es extremadamente limitado. ${ }^{16}$

Lo que esta ampliación del concepto de poder hace posible es el considerar tanto su dimensión 'institucional', esto 
es, como un marco socialmente establecido que regula y organiza la conducta, las actitudes, creencias y valores que se aceptan como parte de una cultura: un poder encarnado en prácticas y creencias; cuanto la dimension interpersonal que se manifiesta en formas de conducta 'estratégica': 'dirigir' la conducta de otro o conducirlas para conseguir nuestros fines. Pero estos dos ámbitos, que se entrecruzan de diversas maneras, no están rígidamente fijados y pueden ser transformados. En el nivel de formas de ejercicio del poder entre sujetos, y aun a pesar de las posiciones de ventaja social de las que puedan gozar algunos de ellos, estas relaciones pueden subvertirse, alterarse o invertirse. En el nivel de lo que recibimos como reglas sociales o códigos de conducta (y de valores o códigos morales) caben también actitudes de 'resistencia' por parte de los individuos. De manera que lo que Foucault construye como la dimensión propiamente ética, en particular en la modernidad, es aquella que conforma cada sujeto en el ejercicio de su libertad.

Como es sabido, Foucault distingue entre formas de moralidad, como la de la antigüedad clásica, que le interesa rescatar y reformular, que enfatizan la relación del individuo consigo mismo y con una forma de vivir (que se orienta por tanto a prácticas, hábitos y formas de ascesis para conseguir llegar a ser aquello que se propone como ideal de vida), y moralidades, como la de la edad moderna, que consisten en códigos de normas de conducta que adoptan un modelo legal o jurídico: esto es, toman la forma de leyes válidas para todos y que, como tales, hacen abstracción de las diferencias individuales. Pero el que Foucault quiera advocar la recuperación de una ética como Aphrodisia (o como una 'estética' de la vida) signifi- ca sólo una forma de 'atenuar' el poder (o el ejercicio de algunas prácticas de control y vigilancia sobre los individuos) pero de ninguna manera su eliminación o neutralización. Si consideramos las proposiciones respecto del poder que presentó en el primer volumen de la Historia de la sexualidad ${ }^{17}$ podemos constatar el carácter fluido y móvil que atribuye a las relaciones de poder: ahí aparecen caracterizadas como relaciones de fuerza inmanentes a intercambios no igualitarios (económicos, sexuales y otros) que pueden ser tanto acciones intencionales como no-subjetivas (o resultado de organizaciones institucionales). Son además 'productivas' en tanto que pueden generar cambios e inversiones en la situación de poder. Pero, sobre todo, estas relaciones de poder son vistas como regidas por una dinámica interna que establece las relaciones entre poder/conocimiento/placer.

La idea de 'resistencia' juega un papel clave en esta dinámica, nos dice Foucault: "Cuando hay poder hay resistencia, sin embargo, o precisamente por eso, la resistencia no está nunca en posición de exterioridad con relación al poder". ${ }^{18}$ Las relaciones de poder son estrictamente 'relacionales', y la resistencia no es tampoco monolitica, sino que opera en diversos puntos o momentos de la relación y juega diversos papeles frente a ésta: puede ser adversario, objetivo, apoyo o soporte del poder. Hay, en otras palabras, una 'pluralidad de resistencias', y pueden ser espontáneas $\mathfrak{u}$ organizadas, aisladas o concertadas, pero en cualquier caso, Foucault no les atribuye un sentido único. Esto es, las formas de 'resistencia' al poder no revisten para él necesariamente un carácter moral, si bien éste no queda excluido como posibilidad. 
De hecho, la idea de 'resistencia' a los códigos recibidos es una de las vertientes de la actitud moral tal y como él mismo la define en el segundo volumen de la Historia de la sexua. lidad: "la 'moralidad' se refiere también a la conducta real de los individuos en relación a las reglas y valores que les han sido recomendados; la palabra designa también la manera en que ellos se adhieren de modo más o menos completo a un estándar de conducta, la manera en que obedecen o resisten una prohibición o una prescripción, la manera en que respetan o desatienden un conjunto de valores". 19

Pero la idea de la 'resistencia' al poder de Foucault está revestida de ambigùedad, algunos la han entendido como el nexo que conecta el "poder' con el 'placer'. ${ }^{20}$ La resistencia confronta al poder con el 'otro' ante el cual mide su fuerza y eficacia. La resistencia contribuye a configurar y dar coherencia al poder $y$, aun desde la perspectiva del dominado, ofrece el acceso a placeres perversos (sumision) o compensatorios (venganza) que, no obstante, se ejercen aun desde la pasividad. Habría mucho que decir acerca de los aspectos psicológicos de estos procesos, pero no es éste el lugar para hacerlo. Foucault dedica una buena parte de su obra a escudriñar las tácticas y formas estratégicas de operación de estos mecanismos en la Historia de la sexualidad; lo que me interesa destacar por ahora es solamente un aspecto de ese trabajo: la introducción del 'otro' como ese elemento ambiguo que puede transformar su actitud pasiva de 'resistencia' como contraste al poder, en una actitud activa, y al menos en potencia, de protagonismo moral, dando un sentido diferente a la idea de la 'resistencia'.

Celia Amorós señalaba como una de las tareas éticas del feminismo la de asumir un protagonismo moral completo por parte de las mujeres, una actitud que tendría que llevarnos a criticar prácticas existentes: como el 'doble codigo de moralidad' para hombres $\mathrm{y}$ mujeres, ${ }^{21} \mathrm{y}$ podríamos agregar, la imposición de las condiciones de las relaciones entre los sexos, aun si esto aparece disfrazado de una cierta 'benevolencia' (paternalista) o 'permisividad' que finalmente no resulta tan beneficiosa para las mujeres como se pensaba en los inicios de la llamada 'revolución sexual'. Se trataría de crear un nuevo 'espacio moral' que permitiera condiciones distintas de interacción, esto es, un reajuste de las relaciones de poder que tendiera a corregir asimetrías 'fijadas' como diría Foucault, en arreglos institucionales, en el lenguaje, y en creencias y prácticas profundamente arraigadas. Además del reconocimiento de la dimensión inevitable de 'lucha' de muchas batallas aún por ganar por la actitud de 'resistencia'. He sostenido en otro lugar ${ }^{22}$ que la identidad de las mujeres en nuestra época está atravesada por la idea de la 'resistencia' como consecuencia de nuestra experiencia histórica de exclusión. El feminismo ha sido sin lugar a dudas una de las formas más radicales de cuestionamiento de la tradición, pero además de su parentesco con otras formas de disidencia moral y política, posee un rasgo peculiar: ya que no sólo disiente con respecto de algunas creencias o prácticas que ha recibido de su tradición cultural específica, sino que lo hace de una manera que para ella se considera anómala, es decir, lo hace asumiendo la condición de un sujeto moral autónomo, capaz de 'auto-legislarse".

Además de las batallas políticas aún por librar, el feminismo tiene ante sí 
un vasto territorio de reflexión en cuestiones de filosofía moral. Ideas como las arriba mencionadas de Foucault en torno a las relaciones de poder ofrecen sugerencias interesantes pero tienen también limitaciones importantes. Para concluir, quiera referirme brevemente a una de ellas. Si bien el trabajo del último Foucault respondió a algunos de los cuestionamientos a sus primeras obras -en torno, por ejemplo, al carácter 'ciego' de la reproducción del poder y la ausencia de sujetos- se mantienen dudas importantes respecto de su conceptualización de los aspectos normativos de su teoría del poder (por ejemplo, la crítica de Nancy Fraser ${ }^{23}$ y la sospecha, expresada por Thomas McCarthy, de que el giro 'individualista' de sus últímos trabajos resulta excesivo y suponga nuevas limitaciones, en particular, para articular una teoría política o moral a partir de sus propuestas. ${ }^{24}$

No puedo detenerme más a considerar estos problemas con detalle. Quiero terminar sugiriendo que el introducir la variable de la relación entre los sexos puede indicar un camino a estas reflexiones. Si la crítica al universalismo ético suponía la necesidad de incluir la consideración de las 'diferencias' de los sujetos particulares - no sólo como diferencias en 'estilos' culturales o aún 'morales', sino como asimetrias de poder- conservando sin embargo la idea de un 'protagonismo' moral completo para todos los sujetos humanos, el llevar la reflexión al terreno específico de la relación entre los sexos pone de manifiesto problemas concretos en los intentos de solución de que disponemos.

\section{NOTAS}

1. Charles Taylor, Sources of the Self. The Making of the Modem ldentity, Cambridge Massachusetts, Harvard Univ. Press, 1989.

2. Celia Amorós, Hacia una critica de la razón patriarcal, Barcelona, Anthropos, 1985, p. 122.

3. Carol Gilligan, In a Different Voice: Psychological Theory and Women's Development, Cambridge. Massachusetts, Harvard Univ. Press, 1982.

4. En: Phyllis Rooney, «A Different Voice: On the Feminist Challenge in Moral Theory", The Philosophical Forum, XXII, 4 (1991), p. 340.

5. Seyla Benhabib, "The Generalized and the Concrete Other; the Kohlberg-Gilligan Controversy in Feminist Theory*, Praxis Intemational, 5 , 4 (1986).

6. Celia Amorós, loc. cit, p. 116.

7. Alasdair MacIntyre, After Virtue. A Study in Moral Theory, Notre Dame Indiana, Univ. of Notre Dame Press, 1981, p. 1.

8. Amelia Valcárod, Sexo y Filosofía. Sobre "mujer" y "poder", Barcelona, Anthropos, 1991, p. 11.

9. Michel Foucault, The Use of Pleasure. The History of Sexuality, vol. 2, Nueva York, Pantheon Books, 1985, p. 47.

10. Iris Marion Young, \&Impartiality and the Civic Public: Some Implications of Feminist Critiques of Moral and Political Theoryn, Fentinism as
Critique: Essays on the Politics of Gender in LateCapitalist Societies (compilado por Seyla Benhabib y Drucilla Cornell), Minneapolis, Univ. of Minnesota Press. 1987.

11. Nancy Fraser, aSex, Lies, and the Public Sphere: Some Reflections on the Confirmation of Clarence Thomas\%, Critical Inquiny (The Univ. of Chicago Press) 18 (1992).

12. Jean-François Lyotard, La difenencia, Barcelona, Gedisa, 1991, p. 9.

13. Fraser, loc. cit., p. 596.

14. Lyotard, loc, oit, pp, 175-179.

15. A. Vaícárcel, loc. cit.

16. Citado por Thornas McCarthy "La Crítica de la Razón Impura: Foucault y la Escuela de Francfort», Ideales e IIusiones, Reconstruccion y Desconstrucción en la Teoria Critica Contomporanea (trad. Angel Rivero), Madrid, Tecnos, 1992, p. 73.

17. M. Foucault, The History of Sexuality, vol. 1, Nueva York, Pantheon Books, 1978, pp. 94-95.

18. Ibid, p. 94 (traducción mía).

19. M. Foucault, The History of Sextality. vol, 2, p. 25 (la traducción de todas las citas es mia).

20. Geoffrey Harpharn, *Foucault and the 
Ethics of Power*, Ethics/Aesthetics, Post-Modern Postions (compilada por Robert Merrill), Washington D.C., Maisonneuve Press, 1988, p. 76.

21. Maria Herrera, *Equal Respect Among Unequal Partmers: Gender Difference and the Constitution of Moral Subjects», Phitosophy East and West, A Ouarterly of Comparative Philosophy, XLII, 2 (abril 1992) y en "La identidad como resistencias (no publicado).

23. Nancy Fraser, Unruly Practices, Minneapolis, Univ. of Minnesota Press, 1989.

24. McCarthy, toc. cit., p. 80. 